\title{
Effect of UV irradiation time on the structure and optical properties of Rose Bengal thin films
}

\author{
Zeyada $\mathrm{HM}^{\mathrm{a},{ }^{*}}$, Youssif $\mathrm{MI}^{\mathrm{a}}$, El-Ghamaz NA ${ }^{\mathrm{a}}$, Aboderbala $\mathrm{MEO}^{\mathrm{a}, \mathrm{b}}$ \\ ${ }^{a}$ Department of physics, Faculty of science,34517, University of Damietta. Egypt \\ ${ }^{\mathrm{b}}$ Department of physics, Faculty of science, AlJabl Al Gharbi University, Libya
}

Received: 12 February 2015 / Accepted: 15 March 2015

* Corresponding author: (Fax: +20572403868, Tel.:+20572404097, E-mail address: hzeyada@gmail.com)

\begin{abstract}
Optical properties of pristine and UV irradiated Rose Bengal (RB) films have been investigated using transmittance and reflectance methods. The refractive index $(n)$ and extinction coefficient $(k)$ of RB films were directly calculated from absolute values of transmission and reflection spectra. Single oscillator parameters and the Drude model of free carrier absorption have been used for the analysis of refractive index dispersion. The fundamental absorption edge data were analyzed within the frame work of the band- to-band electron transitions theory. The thickness of RB films has no influence on absorption and refractive indices in the investigated thickness range and within the experimental error. UV irradiation caused a structural transformation of amorphous pristine film to polycrystalline RB film, which resulted in the decrease of absorption coefficient, energy gap and refractive index. The optical functions and their dependence on UV exposure time were directly calculated from the spectral distribution of dielectric constant.
\end{abstract}

Keywords: Rose Bengal; Thin films; UV irradiation; Optical properties; Optical functions

\section{Introduction}

Rose Bengal (2,4,5,7- tetraiodo- 3, '4',5',6'tetrachlofluorescein) is a tetraiodo-substituted dye of the xanthene's class of dyes. It has peculiar photophysical and photochemical properties, such as: high absorption in all solvents in which it is soluble, high intersystem crossing yield and it shows some fluorescence, it is a photodynamic sensitizer, it bleaches slowly in protic, polar solvents and its spectrum is most diagnostic of its immediate environment. Due to the richness in its properties, it has assumed a peculiar role in different fields of disciplines such as applications in bulk heterojunction solar cells (Sharma et al., 2009) and dye- sensitized solar cells (Pradhan et al., 2007; Roy et al., 2008).It is used as a photo sensitizer for the enhancement of conversion efficiency and storage capacity of photo galvanic cells (Gangotri and Bhimwal, 2010), It sensitized $\mathrm{TiO}_{2}$ film electrode (Zhao et al., 1997). Rose Bengal water solutions quenched by potassium iodide can be used to measure instrument response functions of single photon detectors in the orange-red wavelength region (Szabeiski et al., 2009). RB is used as a photo sensitizer in dye 
sensitized photo chemical cells (Jana., 2000; Bahadur and Roy, 1999). And it also has medical and biological applications (Kim et al., 2011; Wang et al., 2013; Chang et al., 2013).

Despite the numerous applications of Rose Bengal dye in various areas, information on the optical and dielectrical properties in bulk and thin film forms is not yet reported. In semiconductor device fabrication materials are usually provided as thin films. In this work, we report for the first time, on the influence of film thickness and ultraviolet exposure time on structure formation, optical and dielectrical properties and optical functions of $\mathrm{RB}$ films prepared by the spin coating technique.

\section{Experimental procedure}

Rose Bengal, RB, $\left(\mathrm{C}_{20} \mathrm{H}_{2} \mathrm{Cl}_{4} \mathrm{I}_{4} \mathrm{Na}_{2} \mathrm{O}_{5}\right)$ was obtained from Aldrich and used as-supplied. The Chemical structure of RB is shown in Fig. 1. RB is dissolved in absolute ethyl alcohol (99.9\%) up to saturation. Homogenous thin films of Rose Bengal (RB) are prepared by the conventional spin coating technique onto pre-cleaned optical flat quartz substrates at room temperature for optical measurements and onto pre-cleaned $\mathrm{KBr}$ substrates for the Fourier transform infrared (FTIR) measurements. Cleaned ordinary glass slides were used as substrates for depositing films for X-ray diffraction (XRD) analysis. The substrates are fixed to the stage of the spin coater and the rotating speed is adjusted to about 2800 $\mathrm{rpm}$. The film thickness was determined optically by the Lloyd's method (Hamza et al., 2003).The thickness of the produced thin films is measured and found to be in the range $110-500 \mathrm{~nm}$. The samples were irradiated with a UV lamp (ColeParmer model 9815-series Lamps 100 watts). The instrument operates with a UV output at $254 \mathrm{~nm}$. FTIR, spectra were recorded using Perkin-Elmer 1340 spectrophotometer. Structural analysis of the powder and thin films is performed at room temperature using $\mathrm{X}$-ray diffraction system (Philips $X^{\prime}$ pert) with filtered $\mathrm{CuK}_{\alpha}$-radiation $(\lambda$ $=1.5405 \AA$ ). The diffraction system was used to investigate the formed phase in the angle range $4-60^{\circ}$.The applied voltage and tube current were $40 \mathrm{kV}$ and $30 \mathrm{~mA}$, respectively. A double beam spectrophotometer (JASCO model V-570$\mathrm{UV} /$ Vis./NIR, Japan) was used for measuring the transmittance, $T(\lambda)$, and reflectance, $R(\lambda)$, of thin films in the wavelength range $200-2500 \mathrm{~nm}$. The measurements were carried out at room temperature and the light beam was adjusted nearly at normal incidence. The absolute values of $T(\lambda)$ and $R(\lambda)$ were used to calculate the optical constants according to the relations (ElNahaas et al., 2002).

$$
\begin{aligned}
& T(\lambda)=\left(\frac{I_{f t}}{I_{q}}\right)\left(1-R_{q}\right), \\
& R(\lambda)=\left(\frac{I_{f r}}{I_{A l}}\right) R_{A l}\left[1+\left(1-R_{q}\right)^{2}\right]-T^{2} R_{q},
\end{aligned}
$$

Where $I_{f t}$ and $I_{q}$ are the intensities of light passing through film quartz system and reference quartz substrate, respectively. The intensities of the light reflected from the sample and from the reference mirror are $\mathrm{I}_{\mathrm{fr}}$ and $I_{\mathrm{Al}}$, respectively. The reflectance of $\mathrm{Al}$ mirror is $R_{A l}$ and $R_{q}$ is the reflectance of the quartz substrate.

The refractive index, $n$, absorption index, $k$, and absorption coefficient, $\alpha$, can be calculated using the absolute values $\mathrm{R}(\lambda)$ and $T(\lambda)$ according to the following relations (Zeyada et al., 2012).

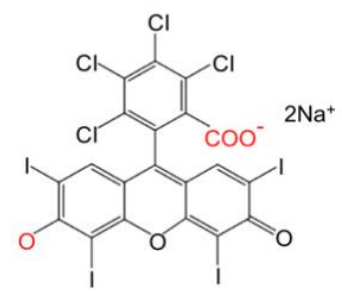

Fig 1. The molecular structure of Rose Bengal.

$$
\begin{gathered}
\alpha=\left(\frac{1}{d}\right) \ln \left[\frac{(1-R)^{2}}{2 T}+\sqrt{\frac{(1-R)^{4}}{4 T^{2}}+R^{2}}\right] \\
k=\frac{\alpha \lambda}{4 \pi} \\
n=\frac{(1+R)}{(1-R)}+\sqrt{\left(\left(\frac{4 R}{(1-R)^{2}}\right)-k^{2}\right)}
\end{gathered}
$$

Where $d$ is the thickness of the film and $\lambda$ is the wavelength of the incident light on the sample. The refractive index, $n$, can be calculated from the relation (El-Nahass and Youssef, 2010).

Since the thickness of the film (d) is previously determined, then the computation can be carried out and the values of $n, k$ and $\alpha$ can be obtained. The experimental errors are taken into account as follows: $\pm 1 \%$ for $\mathrm{T}$ and $\mathrm{R}$ calculations, $\pm 3 \%$ for refractive index, $\pm 2.5 \%$ for 
absorption index and $\pm 2.5 \%$ for film thickness measurements (Konstantinov et al., 1998).

\section{Results and Discussion}

A comparison between the FTIR spectrum of the as received $\mathrm{RB}$ powder, $\mathrm{RB}$ in the as prepared thin film form and UV irradiated thin film condition is shown in Fig. 2. The absorption peaks are assigned and listed in Table 1. In all conditions, there are small shifts in the peak position of the absorption peaks and this does not prevent us from concluding that there is no change in the chemical bonds of RB resulting either from spin coating or irradiation process.

Table 1. Assignment of transmission peaks for RB in powder, pristine and UV irradiated (180 min.) thin films

\begin{tabular}{|c|c|c|c|}
\hline $\bar{v}\left(\mathrm{~cm}^{-1}\right)$ & $\begin{array}{l}\text { Pristine } \\
\text { film } \\
\bar{v}\left(\mathrm{~cm}^{-1}\right)\end{array}$ & $\begin{array}{l}\text { Irradiated } \\
\text { film }(180 \\
\text { min. }) \\
\bar{v}\left(\mathrm{~cm}^{-1}\right)\end{array}$ & Assignment \\
\hline 456.29 & 451.422 & 452.78 & $\mathrm{C}-\mathrm{I}$ \\
\hline 516.33 & 511.46 & 513.64 & $\mathrm{C}-\mathrm{Cl}$ \\
\hline 567.44 & 567.44 & 566.84 & $\mathrm{C}-\mathrm{Cl}$ \\
\hline 614.51 & 614.51 & 617.06 & $\mathrm{C}-\mathrm{Cl}$ \\
\hline 657.51 & 657.51 & 658.57 & $\mathrm{C}-\mathrm{Cl}$ \\
\hline 760.55 & 760.55 & 761.35 & $\mathrm{C}-\mathrm{Cl}$ \\
\hline 952.855 & 952.85 & 953.51 & $\begin{array}{l}\mathrm{C}-\mathrm{C} \\
\text { stretching } \\
\text { vibration }\end{array}$ \\
\hline 1120.81 & 1115.94 & 1118.89 & $\begin{array}{l}\mathrm{C}-\mathrm{H} \text { in } \\
\text { plane bending } \\
\text { vibration }\end{array}$ \\
\hline 1171.2 & 1171.92 & 1169.9 & $\begin{array}{l}\mathrm{C}-\mathrm{H} \text { in } \\
\text { plane bending } \\
\text { vibration }\end{array}$ \\
\hline 1244 & 1240.08 & 1240.42 & $\begin{array}{l}\mathrm{C}-\mathrm{H} \text { in } \\
\text { plane bending } \\
\text { vibration }\end{array}$ \\
\hline 1335.015 & 1339.07 & 1338.51 & $\begin{array}{l}\mathrm{C}=\mathrm{C} \text { stretching } \\
\text { vibration }\end{array}$ \\
\hline 1455.1 & 1450.23 & 1451.67 & $\begin{array}{l}\mathrm{C}=\mathrm{C} \text { stretching } \\
\text { vibration }\end{array}$ \\
\hline 1493.23 & 1489.17 & 1493.18 & $\begin{array}{l}\mathrm{C}=\mathrm{C} \text { stretching } \\
\text { vibration }\end{array}$ \\
\hline 1549.22 & 1553.27 & 1553.78 & $\begin{array}{l}\mathrm{C}=\mathrm{C} \text { stretching } \\
\text { vibration }\end{array}$ \\
\hline 1613.37 & 1617.31 & 1612.36 & $\begin{array}{l}\mathrm{C}=\mathrm{O} \\
\text { stretching } \\
\text { vibration }\end{array}$ \\
\hline
\end{tabular}

The weak peak at $451-456 \mathrm{~cm}^{-1}$ is assigned as $\mathrm{C}-\mathrm{I}$ and the vibration modes in the range 567-760 $\mathrm{cm}^{-1}$ are due to multi $\mathrm{C}-\mathrm{Cl}$ bonds. The absorption peak at $1613-1617 \mathrm{~cm}^{-1}$ is due to $\mathrm{C}=\mathrm{O}$ stretching vibration. The average crystallite size, $D$, can be calculated using the well-known Scherer's equation (Cullity, 1978).

$D=\frac{0.95 \lambda}{\gamma \cos \theta}$

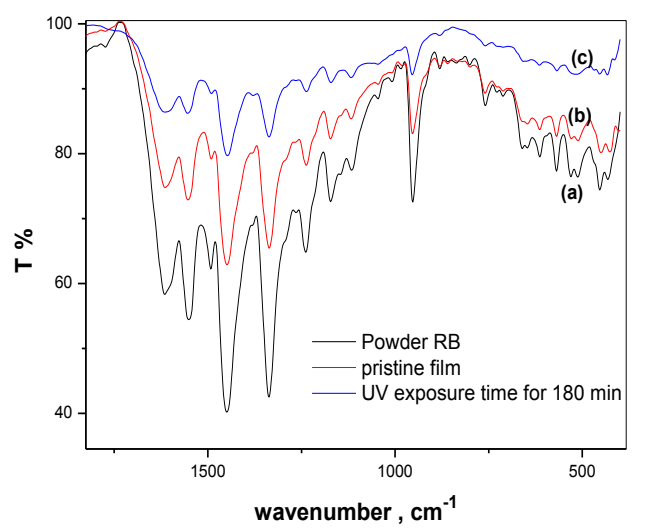

Fig 2. FTIR spectra of RB, (a) powder form, (b) pristine film and (c) UV-irradiated film for $180 \mathrm{~min}$.

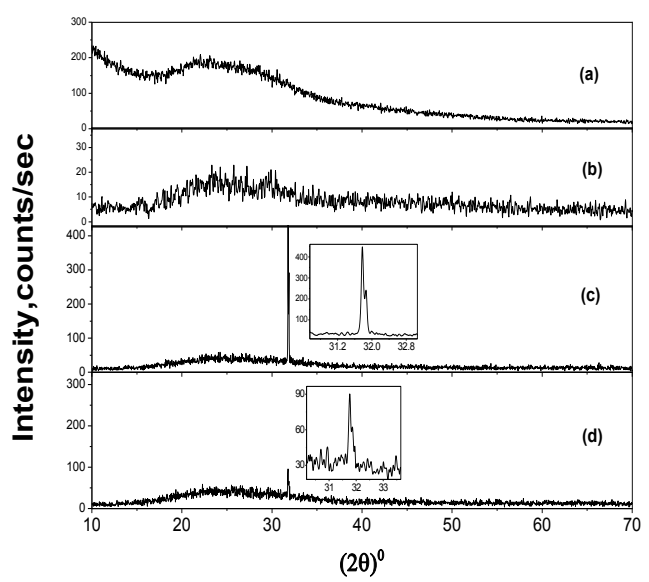

Fig 3. XRD patterns of RB, (a) Powder form, (b) Pristine thin film, (c) UV-irradiated film for $90 \mathrm{~min}$ and (d) UV-irradiated film for $180 \mathrm{~min}$.

Where $\gamma$ the width at the half- maximum peak intensity, measured in radians, $\lambda$ is the $\mathrm{X}$-ray wavelength and $\theta$ is the Bragg's angle. The $\mathrm{x}$-ray diffraction (XRD) patterns of RB in the powder from, pristine and UV-irradiated thin films (exposure times are 90 and $180 \mathrm{~min}$ ) are shown in Fig. 3. As can be observed from Fig. 3 (a,b), there is a halo around $20 \approx 23^{\circ}$ indicating that both of the powder and the pristine thin films of RB have amorphous structure. Fig. $3 \mathrm{c}$ shows that the amorphous structure of the pristine thin film crystallizes into a single polycrystalline phase upon UV exposure for 90 minutes. Fig. 2 and Table 1 illustrate that the amorphous phase of RB 
film has the same chemical bonds as that of polycrystalline phase and this indicates that polymorphous polycrystallization occurred, where atoms in the disordered state jump to crystal front and those in clusters change their orientation to match the growing crystal and deposit onto the crystal front. The determined crystallite size of RB film exposed to UV irradiation time of 90 minutes is $230 \mathrm{~nm}$. Increasing UV irradiation time to 180 minutes recrystallizes the polycrystalline phase of $\mathrm{RB}$ film (Fig. 3(c)) into smaller grains of about 144 $\mathrm{nm}$ in size as shown in Fig. 3 (d).

\section{Optical properties}

Determination of optical constants of the as prepared $R B$ thin films

The influence of film thickness on spectral behavior of $T(\lambda)$ and $R(\lambda)$ for the pristine RB films is depicted in Fig. 4. The determined film thicknesses are 110, 250 and $500 \mathrm{~nm}$. Inspection of Fig. 4 clearly shows that there is no influence of film thickness on spectral behavior of $T(\lambda)$ and $R(\lambda)$ within the investigated thickness range and within the experimental error. The sum of $T(\lambda)$ and $R(\lambda)$ is approximately equal to unity at $\lambda \geq 600 \mathrm{~nm}$ indicating a non-absorbing region while at wavelengths $\lambda \leq 600 \mathrm{~nm}$, the sum of $T$ $(\lambda)$ and $R(\lambda)$ is much less than unity indicating that the RB is a good absorber of light in this region of the spectrum. We conclude also that light is not dispersed and this indicates that the films are homogenous and optically flat. A transmission band is observed in the wavelength range 272- 496nm indicating that RB films can be used as optical filters.

The optical properties of different materials can be fully characterized by the complex refractive index, $n^{*}$, where $n^{*}=n-i k$. In general, the real part, $n$, is related to the dispersion, while the imaginary part (absorption index), $k$, provides a measure of dissipation rate of the electromagnetic wave in the dielectric medium. The calculation of $n$ and $k$ was carried out for films with different thicknesses ranging from 110 to $500 \mathrm{~nm}$. The values of the refractive and absorption indices for RB thin films are computed using the absolute values of transmittance and reflectance. The results depicted in Fig. 5 represent the average values of $n$ and $k$ for the above mentioned thicknesses at given wavelength. The calculations indicated that the optical constants $(n, k)$ do not depend on film thickness of RB films. This result has been observed for some organic semiconductor materials (El-Menyawy et al., 2013; Zeyada et al., 2012; El-Nahass et al., 2004).

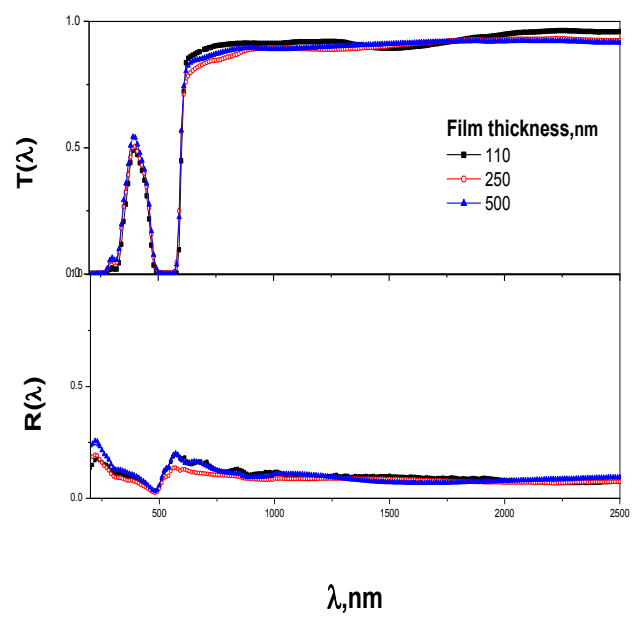

Fig 4. Spectral distribution of transmittance, $T($ ), and reflectance, $\mathrm{R}$ ( ) for the pristine $\mathrm{RB}$ thin films

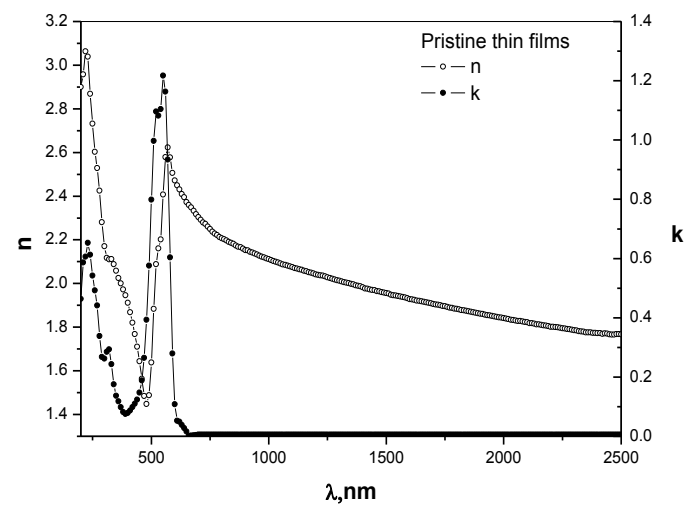

Fig 5. Spectral distribution of refractive index, $n$, and extinction coefficient, $\mathrm{k}$, for pristine RB thin films.

The spectral behavior of $n$ shows a normal dispersion at $\lambda>600 \mathrm{~nm}$ and an anomalous dispersion at $\lambda<600 \mathrm{~nm}$. The dispersion curve (Fig. 5) shows two dispersion peaks and two shoulders in the ultraviolet and visible region of spectra. These features can be explained using the multioscillator model. The spectral behavior of $n$ in the normal dispersion region can be explained by adopting the single oscillator model. In this region, we can deduce optical functions and oscillator parameters at high frequencies from data of refractive index. The results of $k$ show two absorption peaks in the UV region and a doublet peak in the wavelength range $479-650$ $\mathrm{nm}$. The doublet peak in the visible region of 
spectra has been reported for RB by many authors (Sharma et al., 2009; Roy et al., 2008; Pradhan and Pal 2004). All peaks in extinction absorption curve are attributed to the electronic transitions from bonding to anti-bonding molecular orbital (multi oscillator model) (ElKorashy et al., 2003; El-Mallah et al., 2010).

\section{Effects of UV irradiation time on the optical constants of RB thin films}

The effect of UV irradiation time, 90 and 180 min., on the spectral behavior of $T(\lambda)$ and $R(\lambda)$ for the RB thin film of thickness $110 \mathrm{~nm}$ is presented in Fig. 6. Generally, the irradiation process reduces the values of $T$ and $R$ over all the spectrum. No new peaks were observed in the T and $R$ in the absorption region of spectrum. Therefore, UV irradiation has no effect on the transmittance and reflectance properties of the films in the absorption region of the spectrum.

\section{Absorption characteristics of films}

The spectral dependence of absorption coefficient, $\alpha$, for UV irradiated RB thin films, in comparison with that of pristine thin films, is illustrated in Fig. 7. It is clear that the pristine RB films have high absorption coefficient $\left(6 \times 10^{5}\right.$ $\mathrm{cm}^{-1}$ at $2.24 \mathrm{eV}$ ) in the UV-visible region of the spectrum. This indicates that the type of electron transition in the RB films is a direct one. UV irradiation process reduces the value of $\alpha$ all over the spectrum. The decrease of $\alpha$ upon UV irradiation can be attributed to the change in the microstructure of RB film from amorphous, for as-prepared films, to polycrystalline structure for irradiated films as shown in Fig. 3. The observed increase in crystallite size for UV irradiated film for 90 minutes decreases its absorbance, this result is in good agreement with previous work of Hindeleh, et al. (1990).

The transmittance and absorbance of a liquid depends on the molar concentration, $\mathrm{C}_{\mathrm{M}}$, light path length in centimeters, L, and molar absorptivity, $\xi_{\text {molar }}$ for the dissolved solution (Dean, 1992).

$$
\mathrm{A}_{\lambda}=\xi_{\text {molar }} \mathrm{C}_{\mathrm{M}} \mathrm{L}
$$

Beer's law states that molar absorptivity is constant and the absorbance is proportional to the concentration of the dissolved substance at the specified wavelength $(\lambda)$ (Weast, 1975). The molar absorptivity is commonly called molar extinction coefficient and it has units of $\mathrm{mol}^{-1}$ $\mathrm{cm}^{-1}$. The molar extinction coefficient, $\xi_{\text {molar }}$, is a measure of the ability of a molecule to absorb light in non-solid molecular media, can be obtained in terms of the absorption coefficient. If a solid has a concentration of $\mathrm{N}$ molecules per unit volume then $\xi_{\text {molar }}$ can be written as (Schechtman and Spicer, 1970).

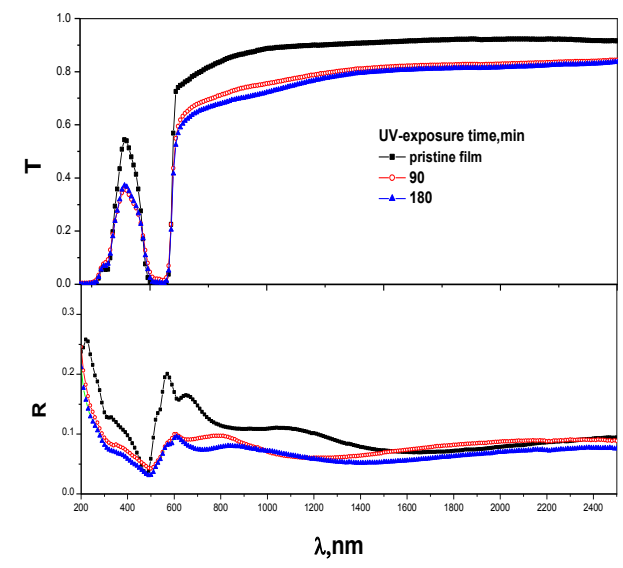

Fig 6. Spectral distribution of transmittance, $\mathrm{T}(\mathrm{)})$ and reflectance, $\mathrm{R}$ ( ) for UV irradiated RB thin films with different exposure times.

$$
\xi_{\text {molar }}=\frac{10^{-3}}{\operatorname{Ln} 10} \frac{N_{a}}{N}=\frac{10^{-3}}{\operatorname{Ln} 10} \frac{M_{W}}{\rho} \alpha=G \alpha
$$

Where $N_{a}$ is Avogadro's number, $M_{w}$ is RB molecular weight, $\rho$ is the material density and $\mathrm{G}$ is a constant. The molar extinction coefficient spectra for the as-prepared and UV-irradiated RB thin films, with a thickness of $110 \mathrm{~nm}$ are depicted in Fig. 8. The as-prepared film exhibited high molar extinction coefficient and the major peak is split into two peaks $\left(B_{x}\right.$ and $\left.B_{y}\right)$.The splitting of the band depends on the distance between the molecules, the angle of the transition dipole moments between neighboring molecules and number of interacting molecules (Schomaker et al., 1959). Fig. 8 shows that the value of $\xi_{\text {molar }}$ decreases with increasing UV exposure time. The peaks $B_{x}$ and $B_{y}$ exchange their peak intensity when UV exposure times exceeds 20 minutes. The remarkable high molar extinction coefficient of $5.4 \times 10^{4}(\mathrm{~mol} . \mathrm{cm})^{-1}$ at wave number $1.9 \times 10^{4} \mathrm{~cm}^{-1}$ for $\mathrm{RB}$ films is comparative with those of other organic dyes (Zeyada et al., 2012; Jiang et al., 2008). High 
molar extinction coefficient and broad absorption band are required in materials to increase the photocurrent density and improve the solar conversion efficiency.

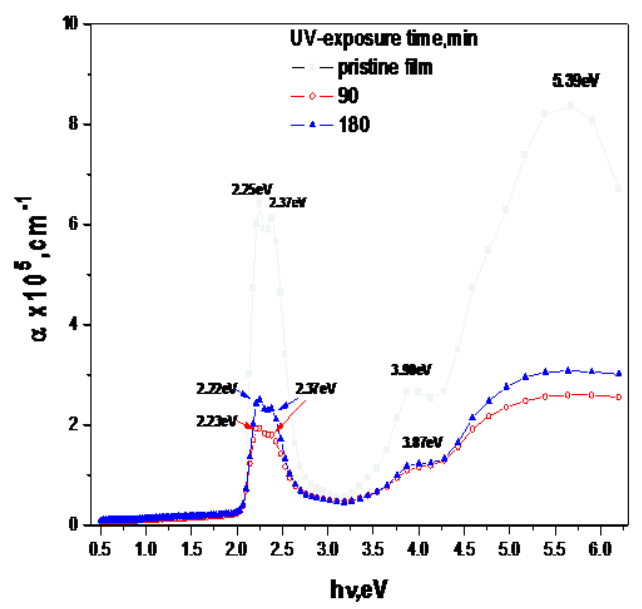

Fig 7. Spectral distribution of absorption coefficient, of RB thin films with different exposure times to UV irradiation.

The molar extinction coefficient spectrum usually allows calculating two spectral parameters, namely the oscillator strength, $\mathrm{f}$, and the electric dipole strength, $q^{2}$, using the following expressions (Kumar et al., 2000).

$f=4.32 \times 10^{-9} \int \xi_{\text {molar }}(v) d v$

and

$q^{2}=\frac{1}{2500} \xi_{\text {molar }}\left(\frac{\Delta \lambda}{\lambda_{\max }}\right)$

Where $\Delta \lambda$ is the band width at half maximum of the absorption peak and $\lambda_{\max }$ is the wavelength at maximum absorption.

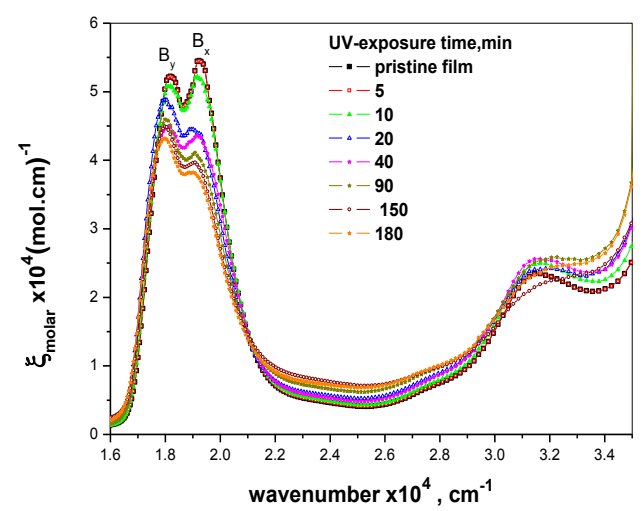

Fig 8. Spectral distribution of molar extinction coefficient for RB thin films with different exposure times to UV irradiation.
The calculated results of oscillator strength, $f$, and electric dipole moment, $q^{2}$, for the asprepared and UV-irradiated RB thin films are listed in Table 2. The two absorption peaks, $B_{x}$ and $\mathrm{B}_{\mathrm{y}}$, are due to splitting in the band and have high intensity, this results in large values of oscillator strengths which are due to parallel electric dipoles (Baerends et al., 2002). Therefore, the increase in spectral parameters is related to the increase of both intensity and broadening of the absorption band. The UV irradiation decreases the intensity of the absorption bands, as shown in Fig. 8, since it rearranges few of electric dipoles from parallel into opposite direction and consequently the oscillator and electric dipole strengths are decreased.

The study of absorption coefficient of a material, at and near the absorption edge, provides valuable information about its band structure. The dependence of absorption coefficient of a material, on the incident photon energy has been formulated by Tauc's relation (Tauc, 1974). As:

$\alpha h v=B\left(h v-E_{g}^{\text {onset }}\right)^{x}$,

Where $B$ is a constant related to the electronic transition probability and $x$ is the power which characterizes the type of transition process, namely $x=1 / 2$ and $3 / 2$ for direct allowed and forbidden transitions, respectively, and $x=2$ and 3 for indirect allowed and forbidden transitions, respectively. The best fit for the data is found for $\mathrm{x}=2$, indicating that the dominant transition is indirect allowed one. Fig. 9 presents the spectral distribution of $(\alpha h v)^{1 / 2}$ as a function of incident photon energy. The value of the energy gap is obtained by extrapolating the straight portion of the curve to intercept the abscissa axis.

Table 2. Parameters of molar extinction coefficient for pristine and UV irradiated RB thin films

\begin{tabular}{ccc}
\hline $\begin{array}{c}\text { UV-irradiation } \\
\text { time, min }\end{array}$ & $f$ & $q^{2}(\AA)^{2}$ \\
\hline Pristine film & 0.82 & 1.448 \\
5 & 0.88 & 1.443 \\
10 & 0.81 & 1.374 \\
20 & 0.73 & 1.193 \\
40 & 0.76 & 1.102 \\
90 & 0.57 & 1.093 \\
150 & 0.49 & 1.062 \\
180 & 0.64 & 1.006 \\
\hline
\end{tabular}

The onset energy gap corresponds to the optical absorption, formation of vacancies, interstials, Frenkel pairs and dislocations in the 
films (Tsiper et al., 2002). The energy gap is the energy gap between HOMO-LUMO bands (Zhokhavets et al., 2003). The values of $E_{g}^{\text {onset }}$ and $E_{g}$ for pristine and UV irradiated RB thin films are tabulated in Table 3. These changes in energy gaps can be attributed to the crystallization process which occurred in RB films upon UV-irradiation as shown previously in Fig. 3. As can be observed from Table 3 and Fig. 9, the UV- irradiation process decreases $E_{g}^{. o n s e t}$ and $E_{g}$. Increasing exposure time of UV irradiation has no influence on energy gap.

Table 3. Dependence of optical and fundamental energy gap of RB films on UV exposure time

\begin{tabular}{lcc}
\hline Exposure time, min. & $E_{g}^{\text {onset }}, \mathrm{eV}$ & $E_{g}^{.}, \mathrm{eV}$ \\
\hline Pristine film & 2.02 & 2.59 \\
90 & 1.98 & 2.33 \\
180 & 1.98 & 2.33 \\
\hline
\end{tabular}

Table 4. Dispersion parameters of pristine and UV irradiated RB thin films

\begin{tabular}{lccccc}
\hline $\begin{array}{l}\text { UV } \\
\text { irradiation } \\
\text { time }\end{array}$ & $\varepsilon_{L}$ & $\varepsilon_{\infty}$ & $\begin{array}{c}\left(\mathrm{N} / \mathrm{m}^{*}\right) \times \\
10^{55}, \\
\mathrm{~m}^{-3} \mathrm{~kg}^{-1}\end{array}$ & $E_{o}(\mathrm{eV})$ & $\begin{array}{l}E_{d} \\
(\mathrm{eV})\end{array}$ \\
\hline Pristine film & 3.613 & 3.272 & 3.325 & 3.091 & 7.024 \\
$90 \mathrm{~min}$ & 3.222 & 2.951 & 2.746 & 3.106 & 6.061 \\
$180 \mathrm{~min}$ & 3.049 & 2.777 & 3.172 & 3.395 & 6.034 \\
\hline
\end{tabular}

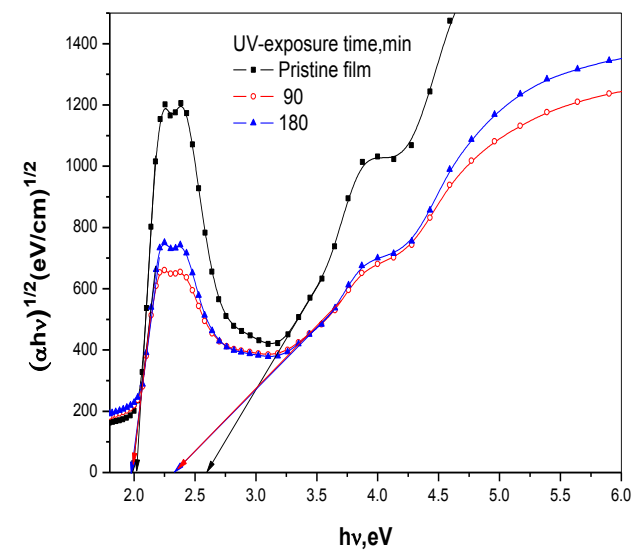

Fig 9. Relation between ( $h$ ) $1 / 2$ and $h$ for RB thin films with different exposure times to UV irradiation.

\section{Dispersion characteristics of RB films}

The spectral distribution of the refractive index, $\mathrm{n}$, for pristine and UV irradiated RB films is illustrated in Fig. 10. It shows an anomalous dispersion in the wavelength range $<600 \mathrm{~nm}$ and exhibits three dispersion peaks and a shoulder for RB films; these are attributed to electronic transition across $\pi-\pi^{*}$ orbital (Davydov, 1971). The normal dispersion is observed for wavelength > $600 \mathrm{~nm}$ and in this wavelength range we can deduce the values of optical functions from the data of refractive index.UV irradiation reduces the value of the refractive index all over the spectra. The decrease of the refractive index with UV irradiation is related to the decrease of mass density of RB films (Andreas et al., 2005). Such behaviour has been reported in other organic semiconductor thin films (El-Nahass et al., 2011; El-Nahass et al., 2012; El-Nahass et al., 2011).

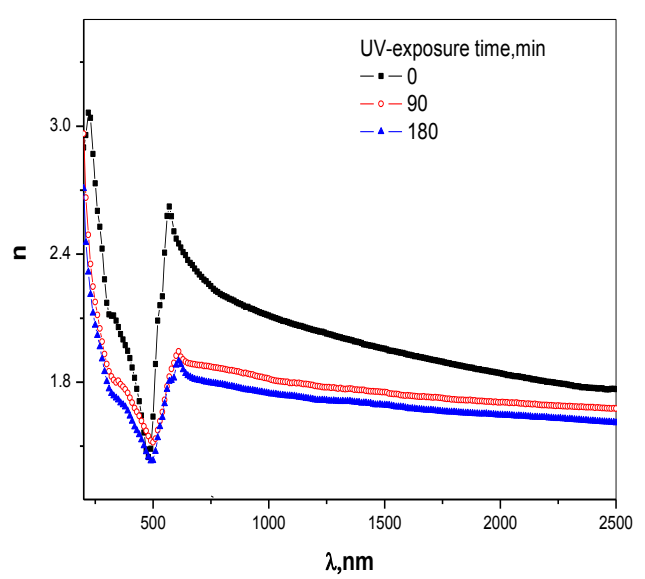

Fig 10. Spectral distribution of refractive index, $n$, for RB thin films with different exposure times to UV irradiation.

Wemple and Di-Domenico (1971) proposed the single oscillator model to explain the spectral dependence of refractive index in the transparent region of spectra, in this model the refractive index is related to the dispersion parameters by:

$$
\frac{1}{n^{2}-1}=\frac{E_{o}}{E_{d}}-\frac{1}{E_{o} E_{d}}(h v)^{2}
$$

Where $h v$ is the photon energy, $E_{o}$ the oscillator energy that gives quantitative information of the overall band structure of the material and $E_{d}$ is the dispersion energy which is a measure of the strength of inter-band optical transition inside the material (Solomon et al., 1988). A plot of $\left(n^{2}-1\right)^{-1}$ versus $(h v)^{2}$ for RB films in the pristine and UV irradiated conditions is illustrated in Fig. 11. The oscillator energy, $E_{o}$, and the dispersion energy, $E_{d}$, are directly 
determined from the slope $\left(E_{o} E_{d}\right)^{-1}$ of the linear portion of the curve and its intercept with ordinate axis $\left(E_{d} / E_{d}\right)$. The values of $E_{o}, E_{d}$ and $\varepsilon_{\infty}$ are listed in Table 4 . and they are influenced by UV-irradiation.

\section{Dielectric characteristics}

Dielectric function relates the electron transitions between bands of a solid to its structure, therefore we can obtain valuable information about the band structure of a solid from its dielectric spectrum. The real, $\varepsilon_{1}$, and the imaginary, $\varepsilon_{2}$, parts of the complex dielectric constant, $\varepsilon^{*}$, are given by(Edward, 1985):

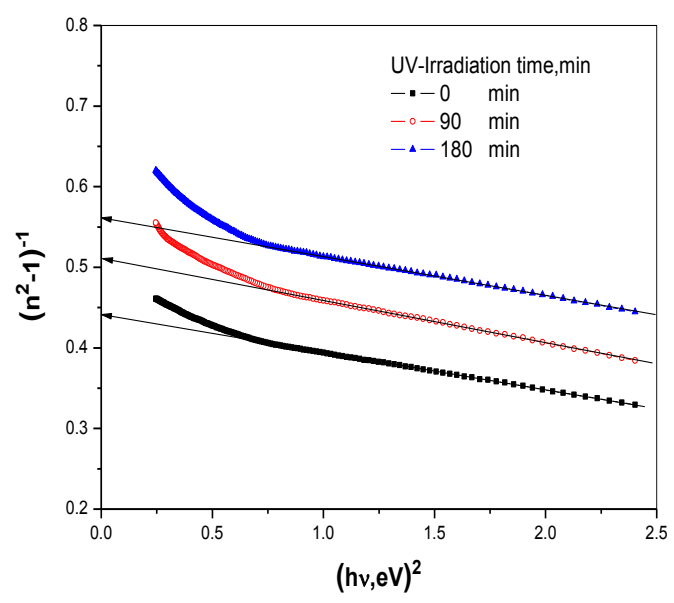

Fig 11. Relation between (n2-1)-1 and (h )2for RB thin films with different exposure times to UV irradiation.

$\varepsilon_{1}=n^{2}-k^{2}=\varepsilon_{L}-\left(\frac{e^{2}\left(N / m^{*}\right)}{4 \pi^{2} c^{2} \varepsilon_{o}}\right) \lambda^{2}$,

$\varepsilon_{2}=2 n k$

where $\varepsilon_{L}$ is the lattice dielectric constant, $\mathrm{N} / \mathrm{m}^{*}$ is the ratio of carrier concentration to the effective mass, $\mathrm{e}$ is the electron charge, $\mathrm{c}$ is the speed of light in vacuum, and $\varepsilon_{o}$ is the permittivity of free space. A graphical representation of $n^{2}$ as a function of $\lambda^{2}$ for the pristine and UV irradiated RB films is shown in Fig. 12. By extrapolating the linear part towards the shorter wavelength, the intercept with the ordinate axis (at $\lambda^{2}=0$ ) gives the value of $\varepsilon_{L}$ and from the slopes of these lines the ratio $N / m^{*}$ can be calculated. The calculated values of $\varepsilon_{L}, \varepsilon_{\infty}$ ,$N / m^{*}$ and the dispersion and oscillator energies are given in Table 4. UV irradiation influences the values of dispersion parameters in comparison to those values of pristine RB film. The value of $\varepsilon_{L}$ differs from the value of $\varepsilon_{\infty}$ and this may be attributed to the structural changes occurred on RB films resulting from UV irradiation. $\mathrm{N} / \mathrm{m}^{*}$ varies with UV exposure times and therefore $\varepsilon_{L}$ and $\varepsilon_{\infty}$ depend on the UV exposure time.

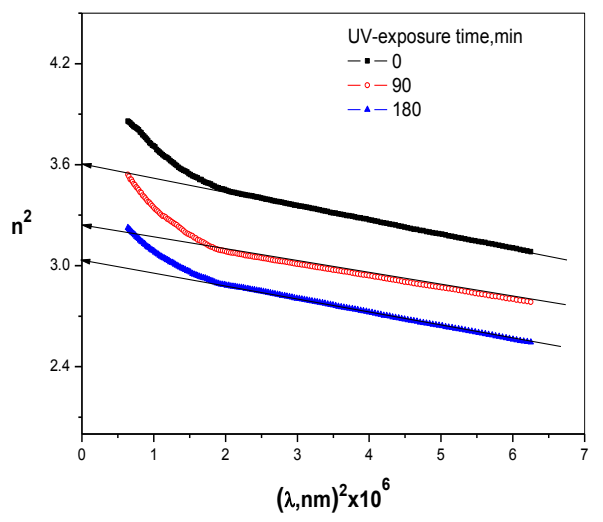

Fig 12. Relation between $n 2$ and for RB thin films with different exposure times to UV irradiation.

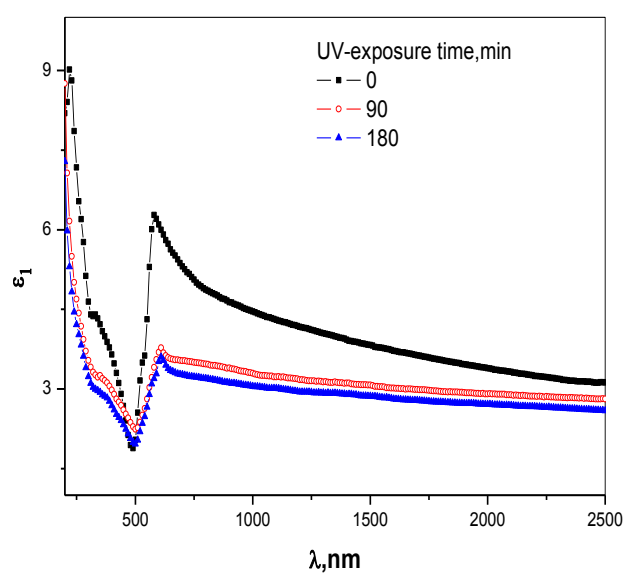

Fig 13. Spectral distribution of for RB thin films with different exposure times to UV irradiation.

The spectral distribution of $\varepsilon_{1}$ and $\varepsilon_{2}$ is shown in Figs. 13 and 14, the values of $\varepsilon_{1}$ are larger than those of $\varepsilon_{2}$ at the same wavelength. The spectral distribution of $\varepsilon_{1}$ and $\varepsilon_{2}$ indicates the presence of different interactions between the incident photons and electrons in the RB film. These interactions affect the shapes of the curves and cause formation of peaks in the dielectric spectra. The decrease in $\varepsilon_{1}$ and $\varepsilon_{2}$ with increasing $\mathrm{UV}$ exposure time is related to 
structural changes in RB films as shown in Fig. 3.

The real $\sigma_{1}$ and the imaginary $\sigma_{2}$ parts of the optical conductivity can be used to detect any further allowed interband optical transitions. The following relation expresses the complex optical conductivity $\sigma^{*}$ in terms of $\sigma_{1}$ and $\sigma_{2}$ (Yakuphanoglu et al., 2004). As:

$$
\begin{aligned}
& \sigma^{*}=\sigma_{1}-\mathrm{i} \sigma_{2}, \\
& \text { where } \\
& \sigma_{1}=\frac{\omega}{4} \varepsilon_{2} \text { and } \sigma_{2}=\frac{\omega}{4}\left(1-\varepsilon_{1}\right)
\end{aligned}
$$

Fig. 15 illustrates the spectral distribution of $\sigma_{1}$ for RB thin films with different exposure times to UV irradiation. Increasing UV exposure time increases the value of $\sigma_{1}$ and this is attributed to variation of crystallite size with $\mathrm{UV}$ exposure time as shown in Fig.3. The onset energy gap is observed at $2.07 \mathrm{eV}$ for pristine and UV irradiated film. The energy gap is observed at $2.85 \mathrm{eV}$ for pristine film and at $2.48 \mathrm{eV}$ for UV exposed film. These results are in agreement with those obtained from Fig.9 and confirm that the type of electron transition is an indirect allowed transition. For photon energy less than $2 \mathrm{eV}, \sigma_{1}$ has a negligible value. UV irradiation has no influence on energy levels of RB but it influenced the onset and energy gaps.

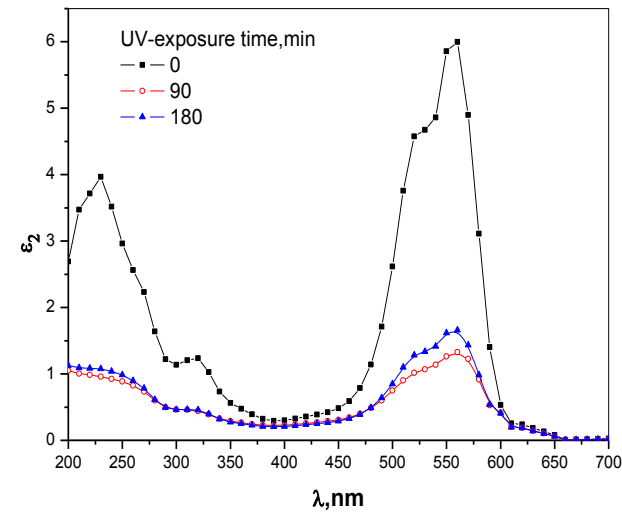

Fig 14. Spectral distribution of for RB thin films with different exposure times to UV irradiation.

Fig. 16 shows the spectral distribution of $\sigma_{2}$ for RB thin films with different exposure times to UV irradiation. Increasing UV exposure time decreases the value of $\sigma_{2}$, this is attributed to microstructure changes in RB films upon UV exposure where it transformed from amorphous structure to polycrystalline as shown in Fig. 3.

Electron- energy-loss spectroscopy has been realized as an important probe for the dielectric response of solids and the intrinsic statistical nature of the particle penetrating phenomenon. The energy loss functions provide a complete description of the response of the material to ionizing particles or photons traveling either through its bulk or on its surface. The surface energy loss, SEL and the volume energy loss, VEL, are related to the related and imaginary parts of the dielectric constant by the following relationships (El- Nahass et al., 2003):

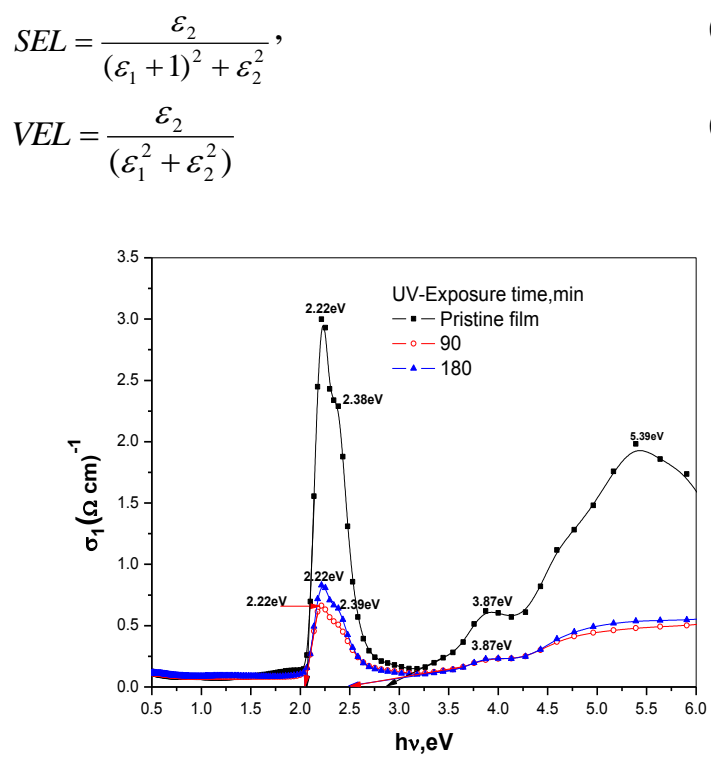

Fig 15. Spectral distribution of $\sigma 1$ for $R B$ thin films with different exposure times to UV irradiation.

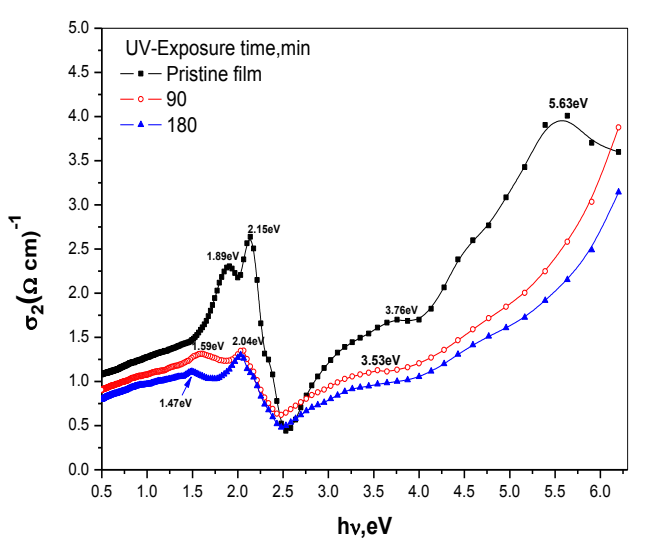

Fig 16. Spectral distribution of $\sigma 2$ for $R B$ thin films with different exposure times to UV irradiation.

Figs. 17 and 18 show the surface energy loss, SEL, and the volume energy loss, VEL, as a 
function of the incident photon energy. Both functions have the same behavior and the energy loss by photons traveling through the material is greater than the energy loss by those passing by its surface. It is clear from these figures that both of VEL and SEL follow the spectral behavior of $\varepsilon_{2}$. The UV-irradiation reduces the values of these quantities all over the spectral region under investigation. The change in their values upon irradiation can be explained in terms of the structural changes shown in Fig. 3.

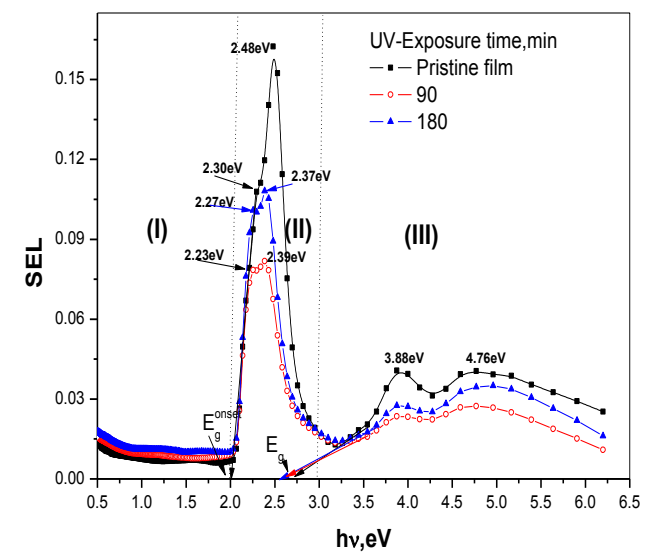

Fig 17. Spectral distribution of surface energy loss for RB thin films with different exposure times to UV irradiation.

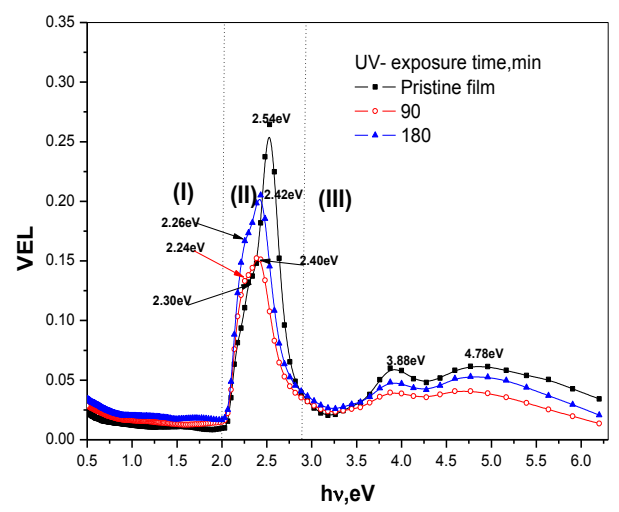

Fig 18. Spectral distribution of volume energy loss for RB thin films with different exposure times to UV irradiation.

The spectra of SEL and VEL can be divided into three regions depending on the incident photon energy: region (I) is for incident photon energy $<2 \mathrm{eV}$, in this region the value of both quantities is near zero and this indicates that there is no interaction between incident photons and $\mathrm{RB}$ films. Region (II) is in the energy range 2-3 $\mathrm{eV}$, at incident photon energy of $2 \mathrm{eV}$ both functions increase rapidly with photon energy and show a shoulder and a peak around 2.3 and $2.5 \mathrm{eV}$, respectively.

These peaks shift a little towards the low energy side of the VEL spectra with the increasing the irradiation time. The shoulder and peak represent vacancies, interstitials, Frenkel pairs and dislocations levels in the energy gap. Region (III) is for incident photon energy $>3 \mathrm{eV}$, both of SEL and VEL show tow peaks close to 3.88 and $4.76 \mathrm{eV}$ represent energy levels in LUMO orbital.

\section{Conclusions}

FTIR analysis for RB films provides an evidence for its chemical stability. RB has an amorphous structure in powder and pristine film forms. Exposure of RB films to UV irradiation resulted in polymorphous polycrystalline of crystallite size that depends on UV exposure time. The data of $T$ and $R$ showed that homogenous optical flat films are obtained by the spin coating technique and these films can be used as optical filters. The results of calculation for $n$ and $k$ showed that they are independent of film thickness in the investigated thickness range of 110-500 nm and within the experimental error. High molar extinction coefficient of $5.4 \times 10^{4}$ (mol.cm) ${ }^{-1}$ and broad band shown in Fig. 8 recommend RB films for application in manufacturing solar cells. The type of electron transition in RB film is indirect allowed transition and the onset and energy gaps are 2.02 and $2.85 \mathrm{eV}$, respectively. UV irradiation decreases the onset and energy gaps to 1.98 and $2.48 \mathrm{eV}$, respectively. Increasing exposure time of UV irradiation has no influence on energy gap. The dispersion parameters of RB films decrease with increasing UV exposure time.

\section{References}

Andreas B, Breunig I, Buse K, (2005) Modeling of Xray-Induced Refractive Index Changes in Poly(methyl methacrylate). Phys. Chem. 6:1.

Baerends EJ, Ricciard G, Rosa A, Van Gisbergen SJ, (2002) A DFT/TDDFT interpretation of the ground and excited states of porphyrin and porphyrazine complexes. Coord. Chem. Rev. 230:5.

Bahadur L, Roy L, (1999) A binary mixture of dyes (2-imidazolin-5-one and Rose Bengal) for photosensitization of $\mathrm{n}-\mathrm{ZnO}$ thin film electrodes in aqueous and acetonitrile media. J. Appl. Electrochem. 29:109-116. 
Chang CC, Yang YT, Yang JC, Wu HD, Tsai T, (2008) Absorption and emission spectral shifts of rose Bengal associated with DMPC liposomes. Dyes and Pigments. 79:170- 175.

Cullity BD, (1978) Elements of X-ray Diffraction. Addison-Wesley Publishing company, Inc.

Davydov AS, (1971) Theory of Molecular Excitons. Plenum press, New York.

Dean JA, Ed., (1992) Langes Handbook of Chemistry, $14^{\text {th }}$ Edition. McGraw- Hill, Inc., New York.

Edward PO, 1985 Hand Book of Optical Constants of Solids, Academic Press, NewYork.

El- Nahass MM, El-Gohary Z, Soliman HS, (2003) Structural and optical studies of thermally evaporated CoPc thin films. Opt. Laser Technol. 35:523-531.

El-Korashy A, El-Zahed H, Radwan M, (2003) Optical studies of $[\mathrm{N}(\mathrm{CH} 3) 4] 2 \mathrm{CoCl} 4$, $[\mathrm{N}(\mathrm{CH} 3) 4] 2 \mathrm{MnCl} 4$ single crystals in the normal para electric phase. Physica B. 334:75.

El-Mallah HM, El-Ghamaz NA and Waly MA,(2010) Influence of UV irradiation on optical properties of thermally evaporated4,4'-(1E,1'E)-2,2'-(2aminopyrimidine-4,6-diyl)bis(ethene-2,1-

diyl)bis(N,N dimethylaniline) thin films. J. Phys. D: Appl. Phys. 43:455407.

El-Menyawy EM, El-Ghamaz NA, Nawar HH,(2013) Infrared spectra, optical constants and semiconductor behavior of 5-(2phenylhydrazono)-3,3-dimethylcyclohexanone thin films. Journal of Molecular Structure. 1036:144-150.

El-Nahaas MM, Zeyada HM, Aziz MS, El-Ghamaz NA (2002) Optical properties of thermally evaporated SnS thin films. Optical Materials.20:159-170.

El-Nahass MM, Abd El-Khalek HM, Nawar AM, (2012) Topological, morphological and optical properties of Gamma irradiated Ni (II) tetraphenyl porphyrin thin films. Optics Communications. 285:1872.

El-Nahass MM, Ammar AH, Atta AA, Farag AAM, El-Zaidia EFM, (2011) Influence of X-ray irradiation on the optical properties of CoMTPP thin films. Optics Communications. 284:2259.

El-Nahass MM, El-Deeb AF, Metwally HS, Hassanien AMH, (2011) Influence of annealing on the optical properties of 5,10,15,20tetraphenyle-21H,23H-porphine iron(III) chloride thin films. Materials Chemistry and Physics 125:247-251.

El-Nahass MM, Youssef TE, (2010) Influence of Xray irradiation on the optical properties of ruthenium (II)octa-(n-hexyl)- phthalocyanine thin film. J. Alloys Compd. 503:86.

El-Nahass MM, Zeyada HM, Hendi AA,(2004) Structural and optical properties of vacuum deposited thin films of (E) $\left[\begin{array}{ll}\alpha & (2,5\end{array}\right.$ dimethylfuryl) ethylidene] (dicyclopropyl methylene) 2,5 furadione. Optical Materials.

\section{$25: 43-52$}

Gangotri KM, Bhimwal MK, (2010) Study the performance of photogalvanic cells for solar energy conversion and storage: Rose Bengal-dXylose-NaLS system. Solar Energy. 84:12941300

Hamza AA, Mabrouk MA, Ramadan Emara WA, AM, (2003) Refractive index and thickness determination of thin-films using Lloyd's interferometer. Optics Communications. 225:341348

Hindeleh AM, Abdallah MA, Braik NS,(1990) Crystallinity Enhances light transmissivity through low density polyethylene sheets. J. of Materials Science. 25:1808- 1812.

Jana AK, (2000) Solar cells based on dyes. J. photochem. photobiol. A, Chem.132:1-17.

Jiang Ke-J, Xia J-b, Masaki N, Noda S, Yanagida S, (2008) Efficient sensitization of nanocrystalline $\mathrm{TiO}_{2}$ films with high molar extinction coefficient ruthenium complex. Inorg. Chim. Acta. 361:783785.

Kim YS, Rubio V, Qi J, Xia R, Shi ZZ, Peterson L, Tung CH, O'Neill. BE, (2011) Cancer treatment using an optically inert Rose Bengal derivative combined with pulsed focused ultrasound Journal of Controlled Release. 156:315-322.

Konstantinov I, Babeva T, Ktova S, (1998) Analysis of errors in thin film optical parameters derived from spectrophotometric measurements at normal light incidence Appl. Opt. 37:4260.

Kumar GA, Thomas J, George N, Kumar BA, krishanan PR., Nampoori VP, Vallabhang CP, (2000) optical absorption studies of free $\left(\mathrm{H}_{2} \mathrm{Pc}\right)$ phthalocyanine doped borate glasses. Phys. Chem. Glasses. 41 (2):89-93.

Pradhan B, Batabyal SK, (2007) Vertically aligned $\mathrm{ZnO}$ nanowire arrays in Rose Bengal-based dyesensitized solar cells. A.-J. Pal, Solar Energy Materials \& Solar Cells 91:769- 773.

Pradhan B, Pal AJ,(2004) Organic heterojunction photovoltaic cells: role of functional groups in electron acceptor materials. Solar Energy Materials \& Solar Cells. 81:469-476.

Roy MS, Balraja P, Kumar M, Sharma GD,(2008) Dye-Sensitized Solar Cell Based on Rose Bengal dye and nanocrystalline TiO. Solar Energy Material \& Solar Cell. 92:909-913.

Roy MS, Balraju P, Kumar M, Sharma GD, (2008) Dye-sensitized solar cell based on Rose Bengal dye and nanocrystalline $\mathrm{TiO} 2$. Solar Energy Materials \& Solar Cells. 92:909- 913

Schechtman BH, Spicer WE, (1970) Near infrared to vacuum ultraviolet absorption spectra and the optical constants of phythalocyanine and porphyrin films. J. Mol. Spectrosc. 33:28-48.

Schomaker V, Waser J, Marsh RE, Bergman G, (1959) To fit a planeor a line to a set of points by least squares. Acta Cryst. 12:600.

Sharma GD, Balraju P, Sharma SK, Roy MS, (2009) 
Charge conduction process and photoelectrical properties of bulk heterojunction device based on sulphonated nickel phthalocyanine and rose Bengal, J. Physics and chemistry of solids. 70:1422-1431.

Sharma GD, Balraju P, Sharma SK, Roy MS, (2009) Charge conduction process and photoelectrical properties of bulk heterojunction device based on sulphonated nickel phthalocyanine and rose Bengal. Journal of Physics and Chemistry of Solids. 70:1422 1431

Solomon I, Schmidt MP, Sénémaud C, Khodja MD, (1988) Band structure of carbonated amorphous silicon studied by optical, photoelectron, and x-ray spectroscopy. Phys. Rev.B. 38:1326.

Szabeiski M, Luchowski R, Gryczynski Z, Kapusta P, Ortmann U, Gryczynski I, (2009) Evaluation of instrument response functions for lifetime imaging detectors using quenched Rose Bengal solutions. Chemical Physics Letters. 471:153-159.

Tauc J, (1974) Amorphous and liquid semiconductors. Plenum press, London.

Tsiper EV, Soos ZG, Gao W, Kahn A, (2002) Electronic polarization at surfaces and thin films of organic molecular crystals: PTCDA. Chem. Phys. Lett. 360:47.

Wang .J.-H, Wang .B, Liu .Q, Li. Q, Huang. H, L.Song, Sun .T.-Y, Wang H, Yu XF, Li C, Chu PK, (2013) Bimodal optical diagnostics of oral cancer based on Rose Bengal conjugated gold nanorod platform. Biomaterials. 34:4274-4283.

Weast RC, Ed ,(1975) Handbook of Chemistry and Physics, $56^{\text {th }}$ Edition. CRC Press, Cleveland.
Wemple SH, DiDomenico M, (1971) Behavior of the Electronic Dielectric Constant in Covalent and Ionic Materials. Phys. Rev. B 3 (4):1338.

Yakuphanoglu F, Sekerci M, Oztuk OF, (2004) The determination of the optical constants of $\mathrm{Cu}$ (II) compound having 1-chloro-2,3- o- cyclohexylidine propane thin film. Opt. Commun.239:275-280.

Zeyada HM, El-Ghamaz NA, Gaml EA, (2013) Effect of substitution group variation on the optical functions of-5-sulfono-7-(4-x phenyl azo)8-hydroxy quinoline thin films. Current Applied Physics. 13:1960-1966.

Zeyada HM, EL-Nahass MM, Elashmawi IS, Habashi AA,(2012) Annealing temperatures induced optical constant variations of methyl Violet 2B thin films manufactured by the spin coating technique. J. Non-Crystalline Solids. 358:625.

Zeyada HM, EL-Nahass MM, Samak SA,(2012) Effect of $\gamma$-ray irradiation on structure formation and optical constants of thermally evaporated rhodamine B thin films. J. Non-Crystalline Solids. 358:915-920.

Zhao G, Kozuka H, Yoko T, (1997) Effect of the incorporation of silver and gold nanoparticles Solar Energy Materials and Solar Cell on the photo anodic properties of rose bengal sensitized $\mathrm{TiO} 2$ film Electrodes prepared by sol-gel method. Solar Energy Materials and Solar Cell. 46:219-231.

Zhokhavets U, Goldhahn R, Gobsch G, Schliefke W, (2003) Dielectric function and one-dimensional description of the absorption of pdy(3octylthioplene). Synthetic Metals. 138:491-495.
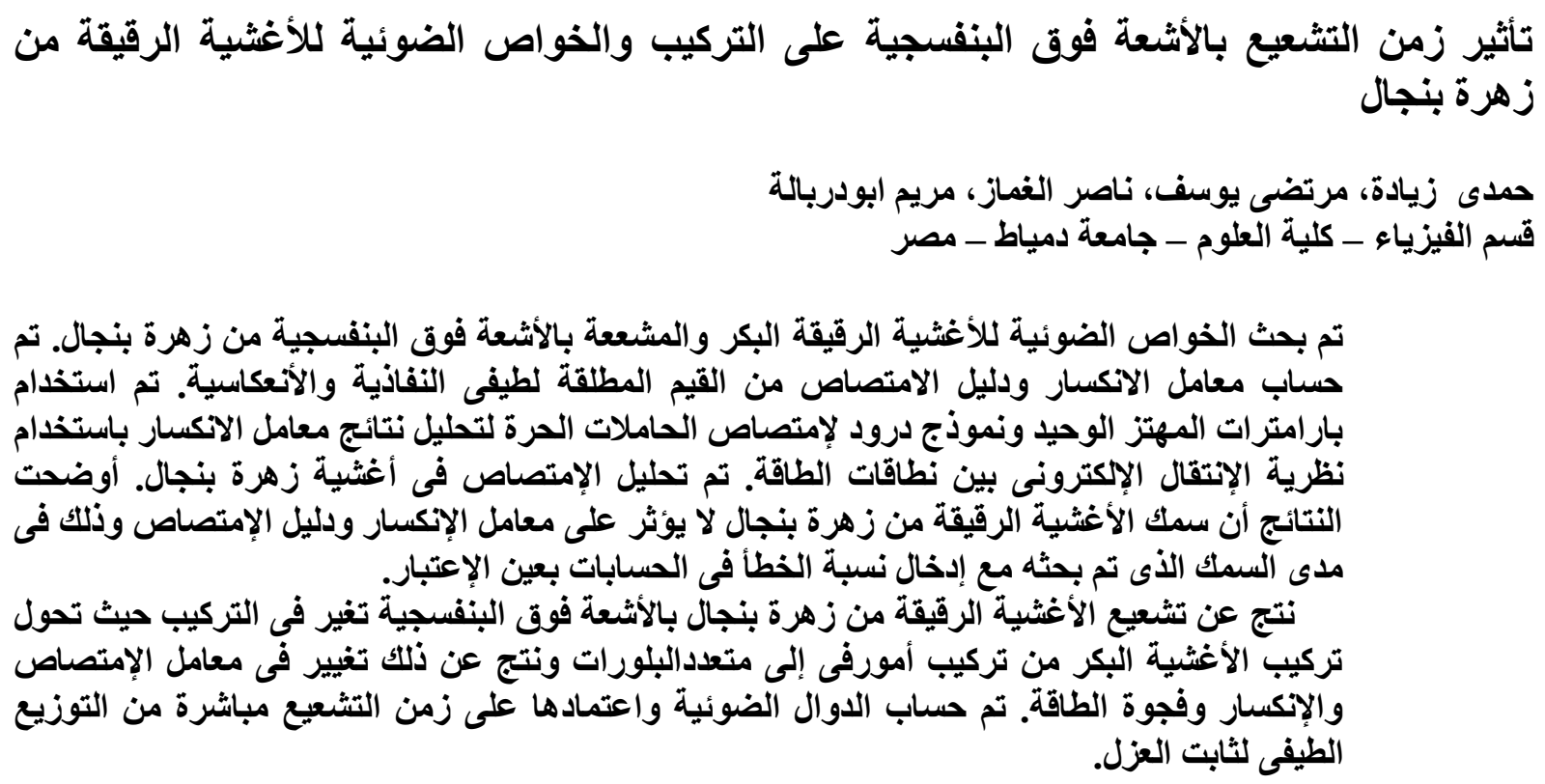